\title{
Les contrats de travail dormants aux Pays-Bas
}

\section{Nicola Gundt}

\section{(2) OpenEdition \\ Journals}

Édition électronique

URL : https://journals.openedition.org/rdctss/1264

DOI : $10.4000 /$ rdctss. 1264

ISSN : 2262-9815

\section{Éditeur}

Centre de droit comparé du travail et de la sécurité sociale

\section{Édition imprimée}

Date de publication : 1 avril 2020

Pagination : 196-199

ISSN : 2117-4350

\section{Référence électronique}

Nicola Gundt, «Les contrats de travail dormants aux Pays-Bas », Revue de droit comparé du travail et de la sécurité sociale [En ligne], 1 | 2020, mis en ligne le 01 novembre 2021, consulté le 11 novembre 2021. URL : http://journals.openedition.org/rdctss/1264; DOI : https://doi.org/10.4000/rdctss. 1264

\section{(c) (1) (9)}

Revue de droit comparé du travail et de la sécurité sociale est mise à disposition selon les termes de la Licence Creative Commons Attribution - Pas d'Utilisation Commerciale - Pas de Modification 4.0 International. 


\section{NICOLA GUNDT}

UNIVERSITÉ DE MAASTRICHT

\section{LES CONTRATS DE TRAVAIL DORMANTS AUX PAYS-BAS}

Un contrat de travail dormant est un contrat qui, bien qu'existant toujours, n'est plus exécuté. L'employeur ne verse pas de salaire et le salarié ne travaille pas. De ce fait, le contrat devient une " coquille vide ». Cette situation posait des difficultés considérables; le législateur comme les tribunaux d'instance furent donc régulièrement sollicités pour tenter de trouver des solutions. En novembre 2019, la Cour suprême s'est finalement engagée sur le sujet. Le présent article examinera la problématique générale des contrats dormants (I), avant d'analyser la jurisprudence des tribunaux d'instance et la réaction du législateur (II), puis d'étudier l'affaire Xella Kalkzandsteenfabriek (III) qui a conduit à l'arrêt de la Cour suprême du 8 novembre 2019 (IV) ${ }^{1}$.

\section{I - PRÉSENTATION DES CONTRATS DE TRAVAIL DORMANTS}

L'existence de contrats de travail dormants est due à deux particularités de la législation néerlandaise sur le licenciement. Tout d'abord, un employeur n'est pas autorisé à licencier un salarié malade durant les 104 premières semaines de congé de maladie ${ }^{2}$. Au contraire, l'employeur doit lui verser un salaire et essayer de le réintégrer dans un emploi approprié . $^{2}$ La deuxième particularité essentielle consiste dans le fait que, selon la loi néerlandaise relative au licenciement, l'employeur devra verser l'indemnité légale de licenciement s'il résilie le contrat de travail ou le fait résilier par un juge. En revanche, si le salarié décide de demander la résiliation de son contrat, l'indemnité légale de licenciement ne sera due que si la résiliation sollicitée par le salarié intervient en réaction à une faute grave de l'employeur, qui revêt dès lors la forme d'un licenciement déguisé4.

Ainsi, l'employeur ayant déjà versé le salaire d'un salarié malade pendant 104 semaines, et ayant aussi la plupart du temps octroyé des frais de réintégration, ne souhaite tout simplement pas résilier le contrat de travail pour éviter de payer lsindemnité légale de licenciement. Le salarié, toujours inapte au travail, reste alors dans l'incertitude, sans droit au salaire après 104 semaines et sans résiliation du contrat. De son côté, l'employeur se retrouve avec un salarié dont la présence sur papier peut avoir des conséquences intéressantes en cas de licenciement collectif ou de licenciement pour raisons économiques.

Les salariés souhaitant obtenir l'indemnité de licenciement ont essayé plusieurs voies à cet effet. En premier lieu, ils ont obtenu la résiliation de leur contrat par un juge. Par la suite, ils ont demandé une attestation selon laquelle le refus de l'employeur de résilier le contrat constituait une faute grave ; de ce fait l'indemnité de licenciement leur était due. Une autre

1 See ECLI:NL:HR:2019:1734 (Affaire Xella Kalkzandsteenfabrieken).

2 Art. 7:670 BW.

3 Art. 7:629 BW.

4 Art. 7:673 BW. 
possibilité consistait à faire valoir que le refus de l'employeur était de mauvaise foi ou, comme on dit aux Pays-Bas : contraire aux obligations d'un «bon employeur $»^{5}$.

\section{II - LA RÉACTION DES TRIBUNAUX ET DU LÉGISLATEUR}

Dans un premier temps, les tribunaux d'instance se sont montrés insensibles à l'argument des salariés selon lequel le fait de ne pas résilier un contrat de travail, tout simplement pour échapper à l'indemnité légale de licenciement, était « injuste ». Néanmoins, l'ancien Ministre des affaires sociales $\mathrm{M}$. Asscher avait pour sa part déclaré que ce comportement n'était pas digne d'un «bon employeur ». Les tribunaux n'ont toutefois pas obtempéré et ont donc refusé toute demande de versement de l'indemnité de licenciement ${ }^{6}$.

Face à cette situation - qui concernait plusieurs milliers de salariés littéralement livrés à eux-mêmes -, le législateur a réagi en adoptant une nouvelle loi : la loi relative à la compensation des indemnités de licenciement. Adoptée en 2018, elle entrera en vigueur le $1^{\mathrm{er}}$ avril $2020^{7}$ et s'appliquera rétroactivement aux contrats résiliés pour cause de maladie de plus de deux ans, à la condition que le salarié ait perdu au moins $80 \%$ de ses capacités de gain et qu'il n'y ait aucune perspective d'amélioration. Cette loi sera financée par le Fonds général pour le chômage, géré par un organisme public, l'UWV, et financée par des primes d'assurance plus élevées pour les employeurs.

La loi relative à l'indemnisation de l'employeur vise à supprimer la principale raison pour laquelle les employeurs préféraient maintenir en sommeil les contrats de travail plutôt que de les résilier. Elle entraîne des modifications dans plusieurs lois et ajoute notamment un article 7:673 au Co de civil qui implique que I'UWV, à la demande de l'employeur, versera une compensation au titre de l'indemnité légale de licenciement due par l'employeur, si le contrat a été résilié pour cause de maladie de longue durée et que le salarié a perdu plus de $80 \%$ de sa capacité de gain. Cette compensation financière est en principe égale à lsindemnité légale de licenciement à laquelle s'ajoutent des frais de reconversion.

Toutefois, même après l'adoption de la loi et en dépit de son caractère rétroactif, les employeurs montraient encore des réticences à mettre fin aux contrats de travail. Mais la loi a conduit des tribunaux d'instance à accepter, soit l'argument selon lequel l'employeur faisait délibérément obstruction, soit celui selon lequel, en tant que bon employeur, il aurait dû résilier le contrat de travail ${ }^{8}$. C'est ainsi que des divergences d'interprétation sont nées entre le juge et au sein de la doctrine juridique sur la bonne voie à suivre?

5 Art. 7:611 BW et jugements de la Cour Suprême.

6 Rb Overijssel, 21 mars 2019, ECLI:NL:RBOVE:2019:1021; Rb Limburg, 3 avril 2019, ECLI:NL:RBLIM: 2019:3068; Rb Limburg, 4 avril 2019, ECLI:NL:RBLIM:2019:3211; Rb Limburg, 30 janvier 2019, ECLI:NL:RBLIM:2019:855; Rb Overijssel, 11 avril 2019, ECLI:NL:RBOVE:2019:1471.

7 Loi du 11 juillet 2018, contenant des mesures relatives à l'indemnité de transition en cas de licenciement pour raisons professionnelles ou invalidité de longue durée (Stb. 2018, 234).

8 Tribunal d'arbitrage des soins de santé 27 décembre 2018, KG 18/24, AR-Updates.n/ 2019-0065.

9 Voirlesjugementsopposés Vzr. in de rechtbankDen Haag, 28 mars 2019, ECLI:NL:RBDHA:2019:3109 versus Vzr. in de rechtbank Gelderland, 29 juillet 2019, ECLI:NL:RBGEL:2019:3440. 


\section{III - L'AFFAIRE XELLA KALKZANDSTEENFABRIEK À L'ORIGINE DE LA QUESTION PRÉJUDICIELLE}

Une affaire dans le sud du pays a offert la possibilité de demander à la Cour suprême de statuer à titre préjudiciel. Dans l'affaire Xella Kalkzandsteenfabriek, un salarié effectuait un travail physiquement exigeant depuis plus de 20 ans, lorsqu'il a commencé à souffrir de maux de dos importants, nécessitant une intervention chirurgicale en 2009. Il a repris ses fonctions en 2010, mais les maux de dos ont réapparu au fil des ans jusqu'à ce qu'en janvier 2016 , il soit de nouveau en incapacité totale de travailler. Son arrêt maladie dure alors plus de 104 semaines d'affilée.

Le taux d'invalidité permanente de ce salarié est alors de 80 à $100 \%$ et, dans la mesure où il n'aura aucune possibilité de reprendre le travail, une pension d'invalidité complète lui est octroyée. A plusieurs reprises dès janvier 2018, ce salarié sollicite auprès de son entreprise Xella Kalkzandsteenfabriek une résiliation de son contrat de travail, une proposition toujours rejetée. Au regard de sa situation privée et matérielle particulièrement difficile - avec une épouse gravement malade, un crédit à la consommation important et une aide régulière de la Banque alimentaire ${ }^{10}$ - le juge de première instance accepte de poser des questions préliminaires à la Cour suprême, s'appuyant, notamment sur les opinions jusque-là contradictoires des tribunaux et l'aspect fondamental des questions juridiques ici en jeu.

Les deux premières questions portent essentiellement sur le droit néerlandais relatif aux modifications du contrat de travail, en vertu duquel un employeur peut faire une offre raisonnable et le salarié doit raisonnablement engager des négociations si les circonstances au travail ont changé ${ }^{11}$. La question consiste désormais à savoir si ce concept peut être inversé : le salarié peut-il demander une modification de son contrat ? Si oui, un changement peut-il aussi signifier un licenciement ? La troisième question s'appuie sur ce point: peut-on soutenir que la persistance d'une incapacité permanente est un changement de circonstances qui justifie une proposition du salarié visant à mettre fin au contrat de travail d'un commun accord? Enfin, si les trois premières questions reçoivent une réponse (même partiellement) négative, l'employeur peut-il néanmoins être tenu d'accepter une résiliation, en particulier lorsqu'une compensation des frais encourus est possible?

\section{IV - LA RÉPONSE DE LA COUR SUPRÊME}

Le 8 novembre 2019, la Cour suprême répond simultanément aux trois premières questions, car elles portent essentiellement toutes sur le fait de savoir si un salarié peut provoquer une modification du contrat, voire une résiliation. Selon la Cour suprême, le principe selon lequel un employeur peut, en raison d'un changement de circonstances au travail, proposer des modifications du contrat de travail, voire engager le licenciement d'un salarié dans le cadre d'une discussion raisonnable, ne peut pas être inversé pour offrir un levier permettant au salarié d'exercer un moyen de pression sur le contrat de travail. La dernière question consiste principalement à savoir si, dans certaines circonstances, le concept de «bon employeur » implique qu'un contrat « dormant » soit résilié - et que

10 ECLI:NL:HR:2019:1734, para 2.2.

11 HR 11 juli 2008, ECLI:NL:HR:2008:BD1847 (Stoof/Mammoet). 
l'indemnité de licenciement soit versée - si le salarié le demande et que l'employeur n'a aucun intérêt raisonnable à ce que le contrat soit prolongé.

La Cour commence par clarifier la manière dont l'indemnité doit être calculée. Le facteur décisif n'est pas le montant que l'employeur peut réclamer, mais l'indemnité de licenciement telle que prévue par la loi. Il peut donc arriver que l'employeur ne soit pas entièrement indemnisé, par exemple si le salaire brut versé est inférieur à l'indemnité légale de licenciement. Le nouvel art. 7:673e BW ne vise pas à couvrir tous les frais, mais simplement à empêcher le cumul des coûts salariaux et des frais de licenciement. Selon la Cour suprême, cet objectif est atteint si le salarié se voit offrir l'indemnité de licenciement qui aurait été due le premier jour où l'employeur aurait pu résilier le contrat de travail, soit 104 semaines +1 jour. Ce raisonnement s'applique indépendamment de la possibilité pour l'employeur de récupérer soit le salaire versé, soit l'indemnité légale de licenciement.

Ceci amène la Cour à répondre à la question concrète de la façon suivante : s'il y a une résiliation du contrat pour cause de maladie de longue durée, en principe l'employeur supposé être un bon employeur - doit accepter une proposition du salarié de mettre fin à la relation de travail par consentement mutuel, en lui accordant l'indemnité légale de licenciement. Cette indemnité de licenciement peut être limitée au montant dû le premier jour où le contrat de travail aurait pu être légalement résilié, c'est-à-dire après 104 semaines et 1 jour. Des exceptions sont possibles si l'employeur a un intérêt raisonnable à maintenir le contrat de travail, par exemple si le salarié peut être réintégré ou si la guérison reste possible. Le fait que le salarié ait presque atteint l'âge de la retraite ne constitue pas un intérêt raisonnable ${ }^{12}$.

En conclusion, l'arrêt, comme la nouvelle loi qui entrera en vigueur le $1^{\text {er }}$ avril 2020, ont clarifié plusieurs points. En premier lieu, un employeur ne pourra plus empêcher le versement de l'indemnité de départ en gardant le silence au terme des 104 semaines de congé de maladie et lorsque le taux d'incapacité permanente du salarié sera d'au moins $80 \%$. L'employeur ne sera pas toujours entièrement remboursé ; en outre, il devra préfinancer l'indemnité puisqu'il ne sera indemnisé qu'une fois la loi entrée en vigueur.

La critique scientifique n'est pas tant dirigée contre le jugement lui-même que contre les conditions préalables sous-jacentes. S'il est vrai que la loi ne fait pas de différence entre les salariés malades et ceux en bonne santé, et que la seule différence réside dans celle entre les retraités et les non-pensionnés, il est encore difficile de concevoir que tous ceux qui sont censés n'avoir aucune possibilité de travailler doivent recevoir une indemnité forfaitaire destinée à les aider à trouver un nouvel emploi. Un moyen plus simple d'éviter le cumul des charges financières pour l'employeur aurait été de refuser le droit à une indemnité de licenciement pour les salariés malades depuis plus de deux ans et qui n'ont plus la possibilité d'exercer un quelconque travail. Tel n'a pas été la volonté du législateur néerlandais.

12 En cas de licenciement en raison de l'âge de la retraite, aucune compensation n'est due, ce qui fait que l'intérêt financier est assez important. 